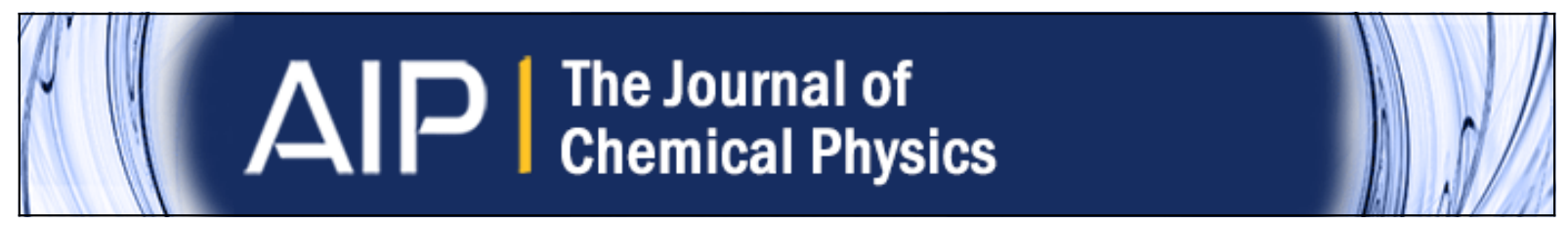

\title{
Computer simulations of localized small polarons in amorphous polyethylene
}

David Cubero and Nicholas Quirke

Citation: The Journal of Chemical Physics 120, 7772 (2004); doi: 10.1063/1.1667471

View online: http://dx.doi.org/10.1063/1.1667471

View Table of Contents: http://scitation.aip.org/content/aip/journal/jcp/120/16?ver=pdfcov

Published by the AIP Publishing

Articles you may be interested in

Vibron-polaron critical localization in a finite size molecular nanowire

J. Chem. Phys. 122, 014701 (2005); 10.1063/1.1828031

Novel method to estimate solubility of small molecules in cis-polyisoprene by molecular dynamics simulations J. Chem. Phys. 115, 6258 (2001); 10.1063/1.1398590

Molecular dynamic simulation of the interaction, at high energy, between the $\mathrm{N} 2$ molecule and polyethylene J. Chem. Phys. 113, 8187 (2000); 10.1063/1.1317228

Clustering of water in polyethylene: A molecular-dynamics simulation

J. Chem. Phys. 109, 6476 (1998); 10.1063/1.477293

Subglass chain dynamics and relaxation in polyethylene: A molecular dynamics simulation study

J. Chem. Phys. 108, 9912 (1998); 10.1063/1.476430

How can you REACH $100 \%$

of researchers at the Top 100

Physical Sciences Universities?

With The Journal of Chemical Physics.

AIP $\left.\right|_{\text {Chemical Physics }} ^{\text {The Journal of }}$

THERE'S POWER IN NUMBERS. Reach the world with AIP Publishing.

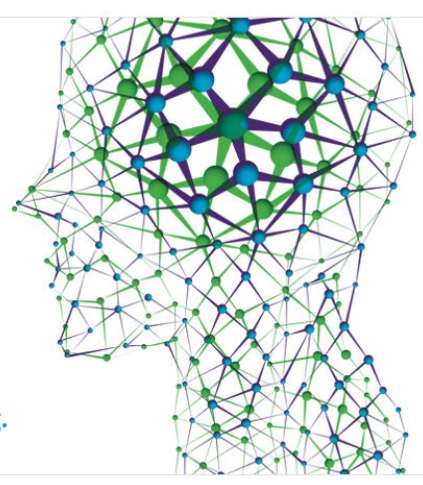




\title{
Computer simulations of localized small polarons in amorphous polyethylene
}

\author{
David Cubero and Nicholas Quirke \\ Department of Chemistry, Imperial College, London, SW7 2AY, United Kingdom
}

(Received 20 October 2003; accepted 14 January 2004)

\begin{abstract}
We use a simple mean field scheme to compute the polarization energy of an excess electron in amorphous polyethylene that allows us to study dynamical properties. Nonadiabatic simulations of an excess electron in amorphous polyethylene at room temperature show the spontaneous formation of localized small polaron states in which the electron is confined in a spherically shaped region with a typical dimension of $5 \AA$. We compute the self-trapping energy to be $-0.06 \pm 0.03 \mathrm{eV}$, with a lifetime on the time scale of a few tens of picoseconds. (C) 2004 American Institute of Physics. [DOI: 10.1063/1.1667471]
\end{abstract}

\section{INTRODUCTION}

Polyethylene is the simplest organic insulator, playing a very important role in a number of technological applications such as high tension insulation. Despite a vast literature ${ }^{1}$ concerned with the experimental characterization of its electrical properties, very little is known about the details of electronic transport at the molecular level. An understanding of the mechanisms of charge transport in these materials is important in determining the electronic and optical properties and in the development of new materials with more reliable insulating and other properties.

Recent femtosecond spectroscopy experiments have shown the existence of shallow self-trapped polarons in ultrathin alkane layers on a silver(111) surface. ${ }^{2}$ In addition, recent Car-Parrinello simulations ${ }^{3}$ have shown the spontaneous formation of self-trapped polarons in bulk crystalline polyethylene (modeled using four chains of seven methylene units in periodic boundary conditions, each one initially alltrans). This shallow polaron has been linked to the formation of two opposite trans-gauche defects in a single chain, with the electron trapped in the rotated portion of the chain. In this paper, we will demonstrate the formation of localized polarons in amorphous polyethylene. As in bulk crystalline polyethylene we find very small polarons, with self-trapping energies comparable with the thermal energy $k_{B} T$, but the geometry of the self-trapped state is very different, being essentially isotropic, reflecting the underling symmetry of the dielectric phase. However, with such small self-trapping energies one could question the physical meaning of such localized polaron states. We will show that the lifetime of these states is large enough to allow them to play a very important role in electron transport.

In our previous work we developed a new pseudopotential for electron-polyethylene interactions, which has been used to study electronic states in crystalline and amorphous configurations of polyethylene. These states are found to be in good agreement with the experimental data. ${ }^{4,5}$ We also have been able to locate the mobility edge (separating localized and extended states) in amorphous polyethylene at about the vacuum level. The localized states extend to about
$-0.3 \mathrm{eV}$. All the simulations presented in this article start from levels below the mobility edge.

The article is organized as follows: in Sec. II we describe the methods and simulation details. In Sec. III we describe the mean-field approach we have used to carry out dynamical simulations. In Sec. IV we describe the self-trapped polaron. Finally, Sec. V provides a short summary and conclusions.

\section{METHODS}

We have used a mixed quantum-classical approach to study the dynamics of an excess electron in amorphous polyethylene at room temperature. The electron is treated quantum-mechanically while each methylene group is treated as a classical particle. A fast Fourier transform block Lanczos diagonalization algorithm ${ }^{6}$ was used to compute the adiabatic electronic states of each classical configuration every time step. The time evolution of the system was generated on a Born-Oppenheimer potential energy surface using the molecular dynamics code DLPOLY. ${ }^{7}$ The Hamiltonian of the classical subsystem contains all the standard ingredients used to simulate polyethylene (bond stretch, valence angles, and dihedrals terms) plus the interaction energy with the excess electron, which is computed using the HellmanFeynman theorem. ${ }^{8}$ Transitions between different electronic energy surfaces are allowed and computed by means of the Tully's fewest switches surface hopping algorithm. ${ }^{9}$

We have simulated polyethylene systems employing a single chain in a cubic box with periodic boundary conditions. The chain was made of $360 \mathrm{CH}_{2}$ units, though we have also performed simulations with a chain of $1215 \mathrm{CH}_{2}$ units for comparison purposes. The initial equilibrium configurations of amorphous polyethylene at room temperature were generated using the procedure described in Ref. 5. The electronic wavefunctions are represented on a grid of $32^{3}$ or $46^{3}$ points, depending on the system size. We estimate the uncertainty in the results due to grid size as $0.02 \mathrm{eV}$. All interactions were truncated at $r_{c}=9 \AA$, with all electronic energies including a long-range correction based on the polarizability interaction. $^{5}$ 


\section{MEAN FIELD APPROACH}

The interaction energy between the excess electron at $\mathbf{r}$ and the $\mathrm{CH}_{2}$ units at $\mathbf{R}_{i}$ can be decomposed into repulsive and attractive parts:

$$
\left.V\left(\mathbf{r}, \mathbf{R}_{i}\right)=\sum_{j} V_{j}^{r}\left(\left|\mathbf{r}-\mathbf{R}_{j}\right|\right)\right)+V^{p}\left(\mathbf{r}, \mathbf{R}_{i}\right),
$$

where $V_{j}^{r}(r)$ is a short-range repulsive pair potential ${ }^{5}$ and $V^{p}$ is the electrostatic energy that accounts for the point chargeinduced-dipole polarization interaction:

$$
V^{p}\left(\mathbf{r}, \mathbf{R}_{i}\right)=-\frac{1}{2} \sum_{j} \mathbf{p}_{j} \cdot \mathbf{E}_{j}^{(0)} S_{j}\left(\left|\mathbf{r}-\mathbf{R}_{j}\right|\right),
$$

with

$$
\mathbf{E}_{j}^{(0)}=-e \frac{\mathbf{R}_{j}-\mathbf{r}}{\left|\mathbf{R}_{j}-\mathbf{r}\right|^{3}}
$$

the direct electric field due to the excess electron, and

$$
\mathbf{p}_{j}=\alpha_{j} \mathbf{E}_{j}
$$

the dipole moment of the united atom $j$ with a polarizability tensor $\alpha_{j} . S_{j}(r)$ is the switching function that vanishes when $r \rightarrow 0$, accounting for the finite size of the methylene units. The local electric field at each atom $\mathbf{E}_{j}$ is the solution of the set of equations

$$
\mathbf{E}_{j}=\mathbf{E}_{j}^{(0)}+\sum_{k \neq j} T\left(\mathbf{R}_{j}, \mathbf{R}_{k}\right) \cdot \alpha_{k} \mathbf{E}_{k}
$$

with $T\left(\mathbf{R}_{j}, \mathbf{R}_{k}\right)=\left(3 \hat{\mathbf{R}}_{j k} \hat{\mathbf{R}}_{j k}-1\right) / R_{j k}^{3}, \quad \mathbf{R}_{j k}=\mathbf{R}_{j}-\mathbf{R}_{k}$, and $\hat{\mathbf{R}}_{j k}$ $=\mathbf{R}_{j k} / R_{j k}$. Knowledge of the local electric field $\mathbf{E}_{j}$ allows one to compute the force exerted on each atom due to the polarization interaction as $\left\langle\phi\left|\mathbf{F}_{j}^{p}\right| \phi\right\rangle$, where $\phi$ is the electronic wavefunction and (Appendix A)

$$
\begin{aligned}
\mathbf{F}_{j}^{p}= & e T\left(\mathbf{r}, \mathbf{R}_{j}\right) \mathbf{p}_{j}+\sum_{k \neq j} \frac{3}{R_{j k}^{4}}\left(\left(\mathbf{p}_{j} \cdot \mathbf{p}_{k}\right) \mathbf{R}_{j k}+\left(\mathbf{p}_{j} \cdot \mathbf{R}_{j k}\right) \mathbf{p}_{k}\right. \\
& \left.+\left(\mathbf{p}_{k} \cdot \mathbf{R}_{j k}\right) \mathbf{p}_{j}-5\left(\mathbf{p}_{j} \cdot \mathbf{R}_{j k}\right)\left(\mathbf{p}_{k} \cdot \mathbf{R}_{j k}\right) \mathbf{R}_{j k}\right) .
\end{aligned}
$$

The first term in (6) is the force due to the direct field created by the electron (which because of Newton's third law is minus the force exerted by the dipole $j$ on the electron) and the second is the result of the interaction with all other induced dipoles. In Refs. 4 and 5 we solved self-consistently the set of equations (5) using an iterative approach for several configurations of bulk polyethylene. However, the calculation is very expensive and utterly prohibitive in a simulation of the dynamics of an excess electron in polyethylene since the interaction energy has to be computed every time step.

In 1967 Lekner proposed $^{10}$ a mean field approach to compute the polarization interaction of a point charge in a system of isotropic dipoles (with a scalar polarizability $\alpha$ ). In this approximation, the local field at each atom is replaced by

$$
\mathbf{E}_{j}=\mathbf{E}_{j}^{(0)} f\left(\left|\mathbf{R}_{j}-\mathbf{r}\right|\right),
$$

where $f(r)$ is a function that accounts for the screening of the direct electric field exerted by the electron at the dipole $j$ due to the other induced dipoles. Assuming (7) and summing the contribution of all induced dipoles in a statistical way, we obtain an equation for $f(r):^{10}$

$$
f(r)=1-\pi n \alpha \int_{0}^{\infty} d s g(s) s^{-2} \int_{|r-s|}^{|r+s|} d t f(t) t^{-2} \theta(r, s, t)
$$

with

$$
\theta(r, s, t)=\frac{3}{2 s^{2}}\left(s^{2}+t^{2}-r^{2}\right)\left(s^{2}+r^{2}-t^{2}\right)+\left(r^{2}+t^{2}-s^{2}\right) .
$$

In Eq. (8) $n$ is the number density and $g(r)$ is the pair correlation function of the dipole system, usually taken as the equilibrium pair correlation function in absence of the excess electron. Strictly speaking $g(r)$ should be modified due to the presence of the electron; however, neglecting this perturbation has been shown to be very successful when applied to simple polarizable fluids. ${ }^{11}$ With this approach $f(r)$ has to be solved numerically using an iterative self-consistent method, but only once and as a result we obtain a pairwise additive interaction energy that can be computed efficiently every time step.

The first difficulty in applying this mean-field approach to polyethylene is that the polarizability for the methylene units is not a scalar but a tensor. In fact we will show later that the mean-field approximation breaks down for strong anisotropy in the polarizability tensor. Another difficulty arises when the system is made of dipoles of different polarizability, which again is our case, since our pseudopotential contains two different polarizability centers: one at the center of the $\mathrm{CH}_{2}$ units (in $\AA^{3}$ ),

$$
\alpha_{\mathrm{CH}_{2}}=\left(\begin{array}{lll}
0.5 & & \\
& 1.12 & \\
& & 1.64
\end{array}\right)
$$

(in a coordinate system along the symmetry axes of the methylene unit ${ }^{5}$ ), and another at the center of the $\mathrm{C}-\mathrm{C}$ bonds

$$
\alpha_{\mathrm{C}-\mathrm{C}}=\left(\begin{array}{lll}
2.103 & & \\
& 0 & \\
& & 0
\end{array}\right)
$$

(along the $\mathrm{C}-\mathrm{C}$ bond).

These complications are difficult to overcome theoretically. We show below, however, that this is not necessary, since a naive application of Lekner's mean-field method to polyethylene already provides good results.

In Fig. 1 we have plotted the screening function $f(r)$ (solid line) that results when we use Eq. (8) with a total average polarizability for the methylene units,

$$
\bar{\alpha}_{\mathrm{CH}_{2}}=\frac{1}{3}\left[\operatorname{Tr}\left(\alpha_{\mathrm{CH}_{2}}\right)+\operatorname{Tr}\left(\alpha_{\mathrm{C}-\mathrm{C}}\right)\right]=1.788 \AA \text { 列 }
$$

and the pair correlation function $g(r)$ of the $\mathrm{CH}_{2}$ units in amorphous $\mathrm{PE}$ at room temperature computed from a molecular dynamics simulations in absence of the excess electron. This radial distribution function only contains the pairs connected by a van der Waals interactions, which is consis- 


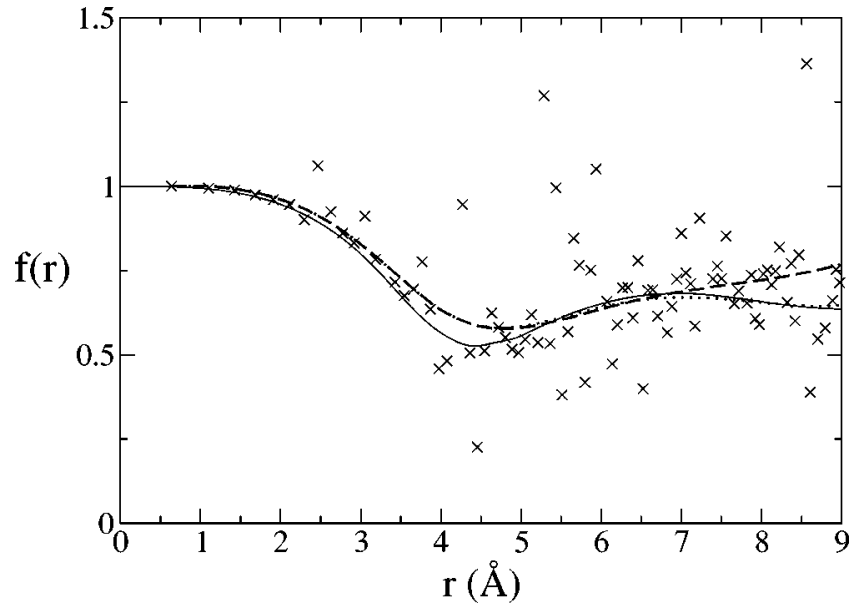

FIG. 1. Screening function $f(r)$. The solid line is the mean-field result. The dash and dotted lines correspond to the screening function for the electron$\mathrm{CH}_{2}$ pair computed from a full many-body simulation with a cutoff radius of $r_{c}=9$ and $12 \AA$, respectively. The crosses correspond to the electron- $(\mathrm{C}-\mathrm{C}$ bond) pairs.

tent with the pseudopotential itself, since the polarizabilities were computed by $a b$ initio methods for alkanes ignoring the intramolecular dipole-dipole interaction. ${ }^{5}$

In order to probe this approximation we have computed the effective screening functions from simulations of amorphous PE at room temperature using the full many-body selfconsistent approach [Eq. (5)], defined for each dipole species as the average over all dipoles of the quantity $\mathbf{p}_{j} \cdot \mathbf{E}_{j}^{(0)} / \mathbf{E}_{j}^{(0)}$ $\cdot \alpha_{j} \cdot \mathbf{E}_{j}^{(0)}$. The results corresponding to a single configuration of a PE chain containing $1215 \mathrm{CH}_{2}$ units are shown in Fig. 1. The screening function of the $\alpha_{\mathrm{CH}_{2}}$ centers (dashed line in Fig. 1) is very smooth, suggesting that a mean field approach would succeed for this type of dipole, where the anisotropy of the polarizability tensor is relatively weak. However, the results for C-C dipoles (crosses) are not smoothly varying and, even though the data seem to be scattered around the $\mathrm{CH}_{2}$ screening function, the dispersion is large enough to reject a mean field treatment.

All screening functions share common features. In the limit $r \rightarrow 0, f(r)$ tends to unity, since the direct Coulomb interaction with the electron becomes dominant, and in the opposite limit $r \rightarrow \infty$ the screening function should approach (in isotropic systems like amorphous PE) the Lorentz local factor $f_{L}=1 /(1+(8 / 3) \pi \bar{\alpha})$ (see, for example, Ref. 12), since at long distances the direct electric field is viewed locally as a constant field. It can be proven that all solutions of the mean field Eq. (8) tend to this limit (see Appendix B), as indeed does our numerical solution of the mean field equation in Fig. 1 (solid line). However, the screening function measured from the full many-body simulation appears to tend to a higher value (dashed line). The reason for this deviation is that the local electric field was computed solving Eq. (5) for all dipoles inside a cutoff sphere of radius $r_{c}$ $=9 \AA$ centered at the excess electron. Since the main contribution to the screening function at each dipole comes from the surrounding dipoles, the screening of the direct electric field due to the dielectric itself is not properly accounted for at dipoles close to the surface of the cutoff sphere. A simu-
TABLE I. Comparison of the energy levels between a full many-body selfconsistent and a mean field calculation.

\begin{tabular}{cccc}
\hline \hline$n$ & $E_{n}^{M B}$ & $E_{n}^{M F}-E_{n}^{M B}$ & $\left\langle\phi_{n}^{M B} \mid \phi_{n}^{M F}\right\rangle$ \\
\hline 0 & -0.224 & 0.148 & 0.996 \\
1 & -0.191 & 0.153 & 0.969 \\
2 & -0.171 & 0.154 & 0.985 \\
3 & -0.148 & 0.139 & 0.972 \\
4 & -0.104 & 0.140 & 0.990 \\
5 & -0.083 & 0.147 & 0.920 \\
6 & -0.036 & 0.138 & 0.198 \\
7 & -0.025 & 0.139 & 0.119 \\
\hline \hline
\end{tabular}

lation of the same amorphous configuration but with a larger cutoff radius $r_{c}=9+3 \AA$ shows that the proper Lorentz limit is recovered (dotted line in Fig. 1). This deviation is of little practical importance, since the wavefunctions of both systems are practically indistinguishable and the error in the energy levels is lower than $0.01 \mathrm{eV}$ (provided the same long range correction based on the Lorentz factor $f_{L}$ is added, ${ }^{5}$ which in this case is as large as $-0.41 \mathrm{eV}$ ).

Apart from the large fluctuations in the $\mathrm{C}-\mathrm{C}$-bond screening function, the full-many body screening functions are in qualitative agreement with the mean field function, with the former clearly higher near the first minimum. This deviation is responsible for an increase in the absolute value of the polarization energy and thus a decrease of the energy levels. In Table I we present a comparison between the energy levels obtained with the full-many body simulation and the mean-field approach using the Lekner's screening function plotted in Fig. 1 for both dipole species and the same configuration. It can be seen that all energy levels are shifted down by approximately the same amount of $0.15 \pm 0.02 \mathrm{eV}$ (with the error taken from our grid resolution error), and that the deviation in the first six wavefunctions is very small (the projection of one normalized eigenstate on the other is close to unity). We have found the same pattern for different configurations and different system sizes.

Since our interest is in the lowest energy levels, we will use this mean-field method in our simulations of the dynamics, correcting all energy levels by $-0.15 \mathrm{eV}$, which can be included in the long-range correction constant. Then, the force exerted by the excess electron in each atom due to the polarization interaction is best computed directly from the interaction energy,

$$
V^{p}=-\frac{1}{2} \sum_{j} \mathbf{E}_{j}^{(0)} \cdot \alpha_{j} \cdot \mathbf{E}_{j}^{(0)} f\left(\left|\mathbf{r}-\mathbf{R}_{j}\right|\right)
$$

[where the switching factor $S(r)$ has been included in the screening factor $f(r)]$. With $\mathbf{F}_{j}^{p}=-\nabla_{j} V^{p}$, where $\nabla_{j}$ is the gradient with respect to $\mathbf{R}_{j}$, we obtain

$$
\mathbf{F}_{j}^{p}=e T\left(\mathbf{r}, \mathbf{R}_{j}\right) \mathbf{p}_{j}+\frac{1}{2}\left(\mathbf{E}_{j}^{(0)} \cdot \alpha_{j} \cdot \mathbf{E}_{j}^{(0)}\right) \nabla_{j} f\left(\left|\mathbf{r}-\mathbf{R}_{j}\right|\right) .
$$

\section{THE LOCALIZED SMALL POLARON}

An excess electron introduced into amorphous polyethylene is expected to interact with the dielectric and be trapped in a distorted region. In Fig. 2 we show the time evolution of the three components of the center of mass and 


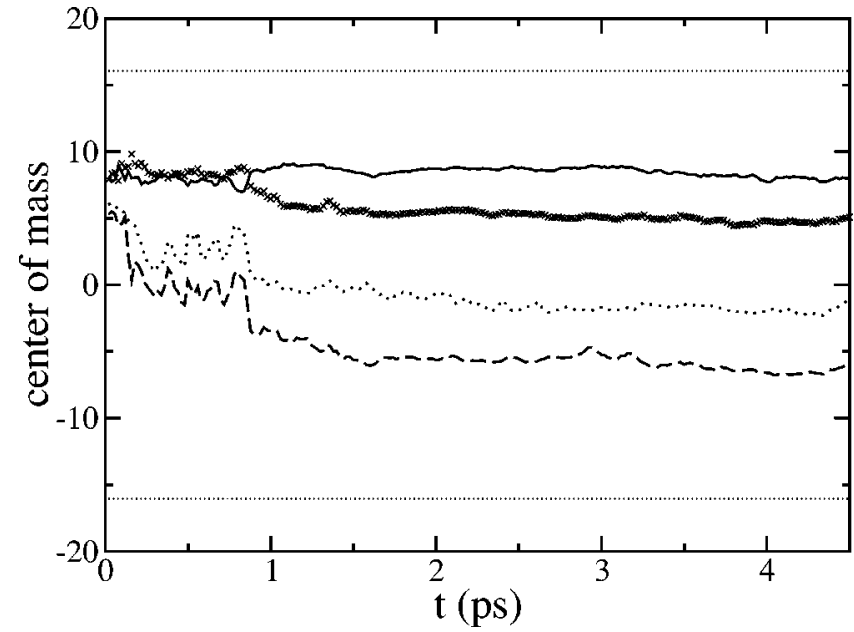

FIG. 2. Components of the excess electron center of mass (lines, in Angstroms) as a function of time from an initially unperturbed configuration of amorphous PE at room temperature. Crosses are the localization length of the electron at each time. System boundary edges are included as a reference (horizontal dotted lines), though the actual position is arbitrary because of the periodic boundary conditions.

the localization length of the excess electron in a microcanonical simulation starting from the ground state of an amorphous PE system of length $L=32.12 \AA$ at room temperature unperturbed by the presence of the excess electron. These quantities were computed under periodic boundary conditions following the procedure sketched in Appendix C. Note that at the beginning the electron is in a state below the mobility edge ${ }^{5}$ and thus localized. However, this state is not stable and the electron explores part of the system, ending up after about $1 \mathrm{ps}$ in a localized state with a smaller length. We present in Fig. 3 the time evolution of the energy spectrum for the same simulation. In this case the electron remains in the ground state, showing an adiabatic relaxation. We have observed similar behavior in all our simulations, though in some cases or if the initial state is not the ground state we witnessed larger relaxation times, of the order of 5-10 ps. In Fig. 4 we show the time evolution of the energy levels in a simulation where the excess electron was initially in the first

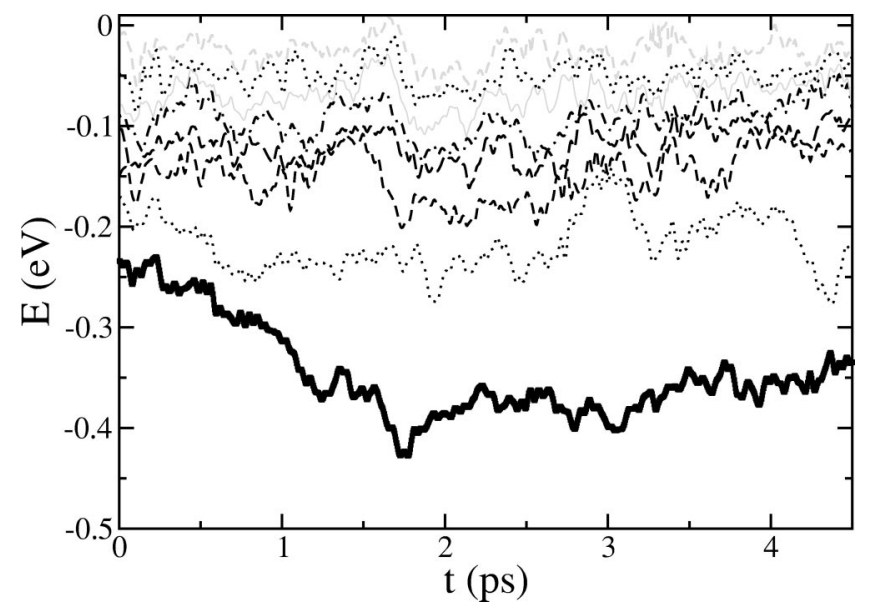

FIG. 3. Electronic energy levels versus time for the same simulation shown in Fig. 2.

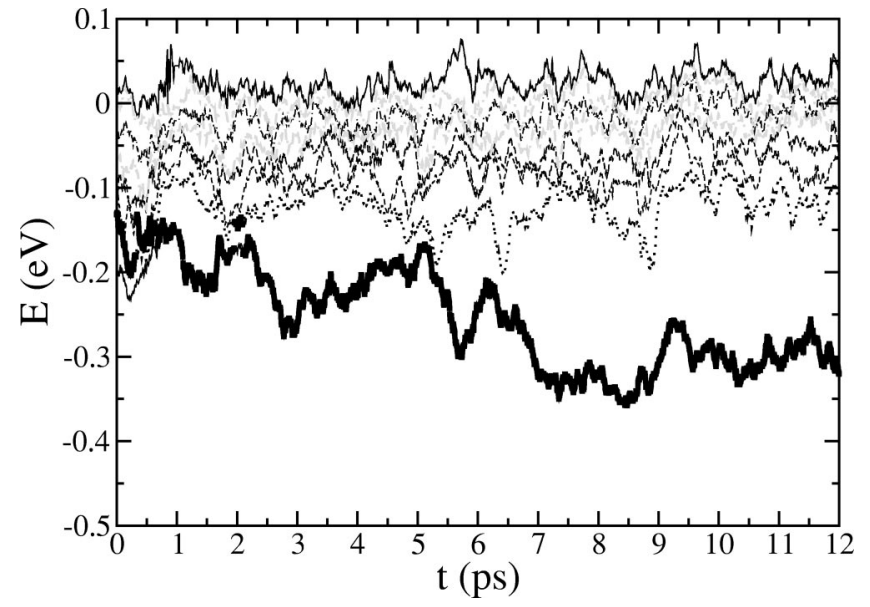

FIG. 4. Electronic energy levels versus time for a simulation starting from an excited state showing a nonadiabatic relaxation. The black squares denote the current energy level.

excited state. The relaxation involves a series of energy hops, indicating a strong nonadiabatic interaction, eventually forming the self-trapped state after about 7 ps.

We present in Fig. 5 the results with a smaller system box of length $L=21.41 \AA$ starting from the ground state. In this case the initial relaxation is not optimally mimicked, since the initial state, though localized, extends through most of the system, but the final self-trapped state is well characterized, showing the same localization length of about $5 \AA$. These smaller systems are much easier to simulate, reducing drastically the computer time used. In the following we will present results computed using this smaller system size.

It is clear from Fig. 3 or 4 that, despite the fact that the kinetic energy is increased due to a reduction of the localization length, the interaction energy becomes more negative. This can only be explained in terms of a smaller repulsion interaction and/or a larger polarization interaction $V^{p}$. This is the reason these states are usually called self-trapping polarons. Part of the energy gained goes to the dielectric to create the distortion that makes possible this polaron state. The difference between the energy gained and the distortion energy (both in absolute value) should be still positive, since

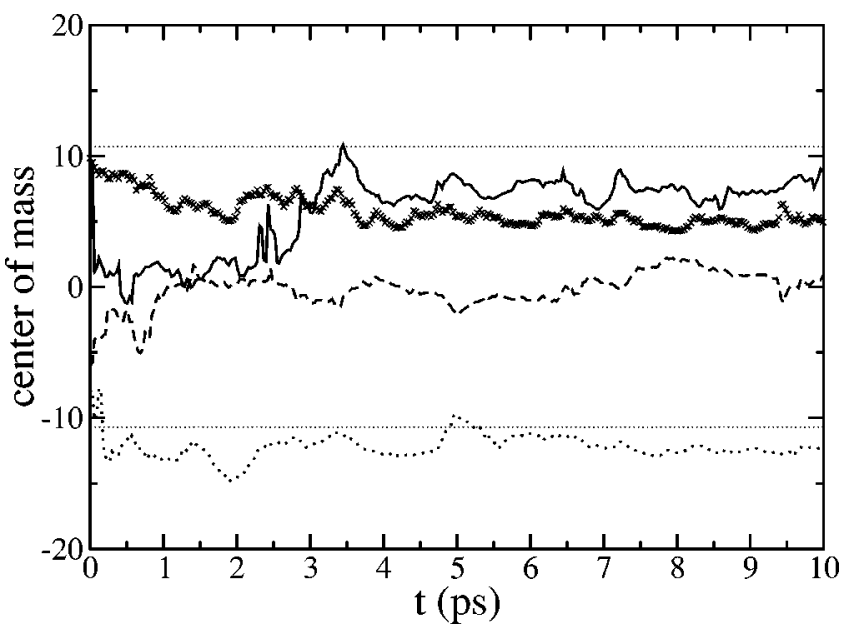

FIG. 5. The same as Fig. 2 but for a simulation with a smaller system size. 
we observe this self-trapped state in every simulation. However, this energy (or more precisely minus this energy), normally called the self-trapping energy, ${ }^{13}$ is difficult to measure from a microcanonical simulation, since the total energy is conserved and thus it is transferred to the dielectric in the form of thermal energy.

In order to compute the self-trapping energy it is more convenient to perform a simulation in the canonical ensemble. We have used the Andersen thermostat, which generates trajectories in the canonical ensemble. ${ }^{14}$ In this case, the quantity that is minimized in the self-trapping process is not the total energy but the free energy $\Delta G_{s t}$. We have computed this free energy using the acceptance ratio method for classical systems, which is described in detail in Ref. 15. To sum up, we need to compute the constant $C$ that satisfies the equation

$$
\sum_{m} h\left(U_{0}-U_{1}+C\right)=\sum_{m^{\prime}} h\left(U_{1}-U_{0}-C\right),
$$

where $h(x)=(1+\exp \beta x)^{-1}$ is the Fermi function $(\beta$ $\left.=1 / k_{B} T\right)$ and $U_{0}$ and $U_{1}$ are the total energies of the classical systems $U_{0}$ and $U_{1}$ at the configurations $m$ (in a simulation of system 1) or $m^{\prime}$ (equilibrium configurations of system 0$)$. The free energy difference $\Delta F$ is then given by

$$
\beta \Delta F=-\ln \left(n_{1} / n_{0}\right)+\beta C,
$$

where $n_{1}$ and $n_{0}$ are the total number of configurations sampled in each system. Since we are interested in the energy difference between the unperturbed ground state and the self-trapped state, we chose $U_{0}$ as the Hamiltonian of the polyethylene system without the excess electron, and $U_{1}$ as the full Hamiltonian with the electron hold at the ground state. Therefore, the self-trapping energy is given by

$$
\beta \Delta G_{s t}=F_{1}-\left(F_{0}+\left\langle E_{0}\right\rangle_{0}\right),
$$

where $\left\langle E_{0}\right\rangle_{0}$ is the average of the electronic ground state energy over the equilibrium configurations of the unperturbed polyethylene system. Averaging over $5 \times 2$ simulations of $10 \mathrm{ps}$ of adiabatic dynamics we obtained $\Delta F$ $=-0.27 \pm 0.01 \mathrm{eV}$ and $\left\langle E_{0}\right\rangle_{0}=-0.21 \pm 0.02 \mathrm{eV}$, which implies $\Delta G_{s t}=-0.06 \pm 0.03 \mathrm{eV}$. Moreover, the averaged localization length of the self-trapped polaron was 5.0 $\pm 0.1 \AA$ and of spherical shape, in agreement with the microcanonical simulations.

It is worth noting that a direct calculation of the selftrapping energy as the total energy difference of the two states (apart from neglecting the entropy change) does not produce an acceptable value due to the large thermal fluctuations of each variable $(\sim 0.1 \mathrm{eV})$. It is much more efficient to use an approach such as the acceptance ratio method, in which the energy difference of both systems is computed for each configuration of both simulations.

We have observed a correlation between the selftrapping polaron and the local density of the dielectric. In all cases we found that the center of mass of the excess electron was within $2 \AA$ of the global minimum of the local atomic density (defined as the number of atomic centers in a sphere of radius equal to the electron localization length divided by the volume of the sphere). Furthermore, this minimum local density was always smaller in the presence of the electron than the minimum local density in the unperturbed system. These changes, which in some systems amounted to a $50 \%$ reduction in local density, clearly indicate the formation of a cavitylike region by means of which the electron lowers its energy.

In order to compare our results in amorphous PE with the self-trapped state found in crystalline $\mathrm{PE}^{3}$ we have monitored the dihedral angle distribution and found no significant difference between the gauche population before and after the polaron is formed, reflecting the fact that in the disordered phase the electron has many more ways to distort the dielectric than in the crystalline phase, in which the electron can only create a cavitylike region by abruptly creating two gauche defects in the otherwise all-trans chains.

In addition, we have observed that the self-trapped polaron is sensitive to the temperature of the system. Simulations at $350 \mathrm{~K}$ show a larger polarization energy, with a smaller localization length of about $4.2 \AA$, while a system at $200 \mathrm{~K}$ shows a more extended excess electron with a typical length of $6.4 \AA$.

The calculations presented above predict that the selftrapping energy is of the same order as the thermal energy at room temperature $\left(k_{B} T=0.026 \mathrm{eV}\right)$, and as a result we would expect rapid trapping and detrapping giving rise to hopping conduction assisted by phonons. This is in fact what we observe in the simulations. In Fig. 6 we present the components of the center of mass of the electron as a function of time for two typical long simulations showing a hop between self-trapping states at different positions. The lifetime of each state is about one order of magnitude larger than the relaxation time to the self-trapping states $(\sim p s)$, demonstrating the physical relevance of these self-trapped states, regardless of their small self-trapping energies. The time evolution of the electronic energy in these simulations shows that this is an adiabatic process, the electron staying in the ground state surface during the hop. The observed lifetime is in agreement with the widely accepted model for adiabatic hopping conduction in polaron theory, ${ }^{16}$ in which the lifetime is estimated as

$$
\tau=\nu^{-1} \exp \left(E_{a} / k_{B} T\right)
$$

with $\nu$ being a typical phonon frequency responsible for the detrapping, and $E_{a}$ an activation energy, here to be identified with the self-trapping free energy $\Delta G_{s t}=-0.06 \pm 0.03 \mathrm{eV}$. Since the optical frequencies in polyethylene are in the range $700-1600 \mathrm{~cm}^{-1},{ }^{17}$ the corresponding phonon energies $h \nu$ are about one order of magnitude larger than $E_{a}$ and thus only the acoustic or torsional (transversal) phonons, with frequencies in the range $0 \leqslant \nu \leqslant 250 \mathrm{~cm}^{-1},{ }^{18}$ can be responsible for the destruction of the self-trapping state. As a result, $\nu^{-1}$ is of the order of a picosecond (or larger), which is about the same time scale for the relaxation to these states, and the exponential factor in (18) provides the factor of 10 observed in the simulations.

Furthermore, this analysis and the simulation results show that the dynamic behavior at the temperature considered is dominated by the low-laying energy states and thus 


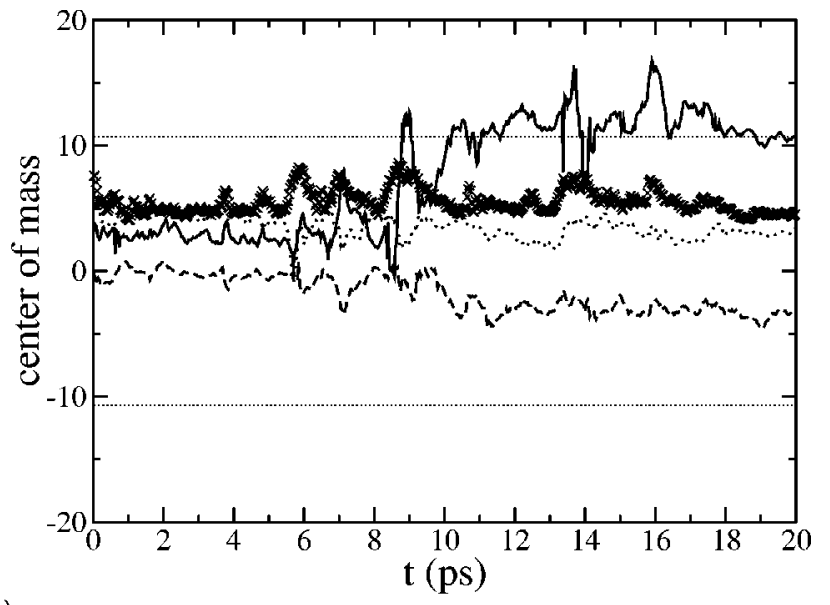

(a)

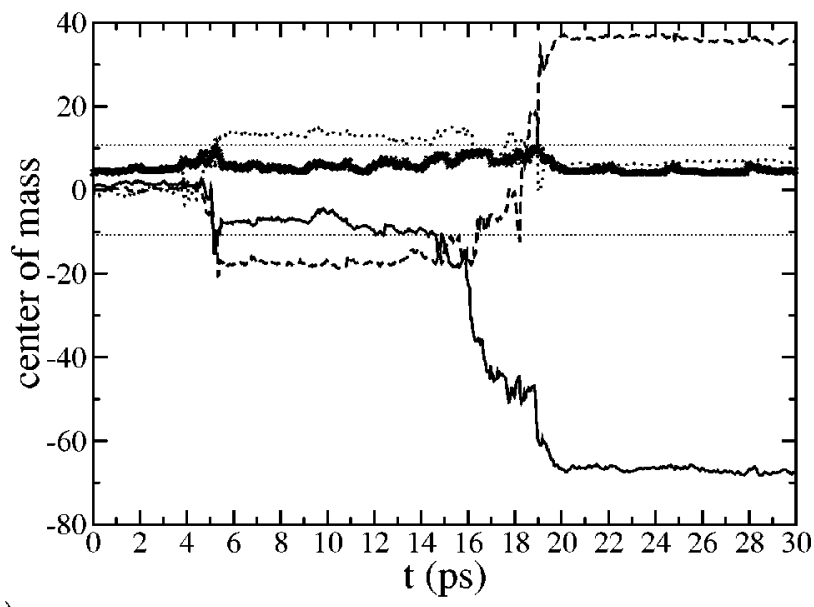

(b)

FIG. 6. The same as Fig. 2 but for two different long simulations showing a hopping mechanism between the self-trapped states.

providing a posteriori justification of the use of the Lekner's mean field method, which describes correctly only the lowest energy states.

\section{CONCLUSIONS}

We have shown that even when a complex nonpolar dielectric like amorphous polyethylene with two species of anisotropic polarizable dipoles is considered, a simple mean field approach neglecting these details produces good results when one is only interested in the lowest energy levels of the excess electron. This method makes dynamical simulations feasible.

Nonadiabatic simulations of an excess electron in amorphous PE at constant energy show the formation of a small self-trapped polaron with a reduced localization length of 5 $\AA$ on picosecond timescales. The wavefunction is isotropic and centered around a small cavitylike region created by the electron. In order to calculate the self-trapping energy we have performed adiabatic simulations in the canonical ensemble using the Andersen thermostat. The self-trapping energy is estimated to be $\Delta G_{s t}=-0.06 \pm 0.03 \mathrm{eV}$ from the free energy change between an unperturbed dielectric system and a system perturbed by the presence of the electron. This is the energy required to remove the self-trapped state and is small when compared to the activation energy for excitation to extended states from unperturbed configurations, i.e., $\sim 0.3 \mathrm{eV}$, thereby providing a justification of the method employed in Ref. 5, in which the effect of the excess electron on the dielectric was neglected. From Ref. 5, we expect electrons thermally excited to energy levels above the mobility edge to provide a contribution to the zero-field mobility of order of $10^{-3} \mathrm{~cm}^{2} / \mathrm{Vs}$, which is in good agreement with the highest values found in experiments.

However, the smallness of the self-trapping energy suggests a hopping mechanism assisted by phonons, which is in fact observed in the simulations. The lifetime of each selftrapping state is observed to be on the time scale of a few tens of picoseconds. We show that this timescale is consistent with an adiabatic model for detrapping and the computed value of the self-trapping energy.

Since the electron is localized and nondegenerate, the corresponding contribution to the mobility can be computed using the Einstein formula ${ }^{13}$ by measuring the diffusion coefficient from very long time simulations. Preliminary calculations show that the contribution to the mobility due to hopping between these self-trapped states may well be about the same order of magnitude as the mobility due to excited electrons above the mobility edge. These simulations are reported elsewhere. ${ }^{19}$

\section{APPENDIX A: POLARIZATION FORCES}

In principle, the force on each dipole $j$ could be computed from the gradient of the polarization energy $V^{p}$. However, it is easier to calculate it from the local electric field. Assume a simple dipole $\mathbf{p}$ at $\mathbf{r}$, then the electrostatic force on the dipole will be

$$
\mathbf{F}=\lim _{d \rightarrow 0, q d \rightarrow p}[-q \mathbf{E}(\mathbf{r})+q \mathbf{E}(\mathbf{r}+\mathbf{d})]=(\mathbf{p} \cdot \nabla) \mathbf{E}(\mathbf{r}) .
$$

If we now consider the full system with dipoles at $\mathbf{R}_{j}$ and an electron at $\mathbf{r}_{0}$, the electric field everywhere is given by

$$
\mathbf{E}(\mathbf{r})=\mathbf{E}^{(0)}(\mathbf{r})+\sum_{k} T\left(\mathbf{r}, \mathbf{R}_{k}\right) \cdot \mathbf{p}_{k},
$$

with $\mathbf{E}^{(0)}(\mathbf{r})=-e\left(\mathbf{r}-\mathbf{r}_{0}\right) /\left|\mathbf{r}-\mathbf{r}_{0}\right|^{3}$. Using these equations, together with

$$
\nabla \mathbf{E}^{(0)}(\mathbf{r})=e T\left(\mathbf{r}, \mathbf{r}_{0}\right)
$$

and

$$
\begin{aligned}
\frac{\partial T\left(\mathbf{r}, \mathbf{r}^{\prime}\right)_{m, n}}{\partial x_{l}}= & \frac{3}{\left|\mathbf{r}-\mathbf{r}^{\prime}\right|^{4}}\left(\frac{\left(x_{l}-x_{l}^{\prime}\right)}{\left|\mathbf{r}-\mathbf{r}^{\prime}\right|} \delta_{m n}\right. \\
& +\frac{\left(x_{m}-x_{m}^{\prime}\right)}{\left|\mathbf{r}-\mathbf{r}^{\prime}\right|} \delta_{l n}+\frac{\left(x_{n}-x_{n}^{\prime}\right)}{\left|\mathbf{r}-\mathbf{r}^{\prime}\right|} \delta_{l m} \\
& \left.-\frac{5\left(x_{l}-x_{l}^{\prime}\right)\left(x_{m}-x_{m}^{\prime}\right)\left(x_{n}-x_{n}^{\prime}\right)}{\left|\mathbf{r}-\mathbf{r}^{\prime}\right|^{3}}\right),
\end{aligned}
$$

we obtain (6). 


\section{APPENDIX B: LONG DISTANCE LIMIT OF THE SCREENING FUNCTION}

The first step to find the long distance limit of the mean field $f(r)$ is to prove

$$
\delta(t-r)=\frac{3}{8} t^{-2} \int_{|t-r|}^{t+r} d s s^{-2} \theta(r, s, t),
$$

where $\theta$ is given by Eq. (9). Since for $t \neq r$

$$
\begin{aligned}
& \int_{|t-r|}^{t+r} d s s^{-2}(r, s, t) \\
& \quad=\left.\frac{(s-r-t)(s+r-t)(s-r+t)(s+r+t)}{2 s^{3}}\right|_{|t-r|} ^{t+r}=0,
\end{aligned}
$$

it is only left to prove that it is properly normalized. Reversing the order of integration

$$
\begin{aligned}
\int_{0}^{t+\varepsilon} d t\left(\frac{3}{8} t^{-2} \int_{|t-r|}^{t+r} d s s^{-2} \theta\right) \\
=\int_{0}^{\varepsilon} d s \int_{|r-s|}^{r+s} d t \square \\
\quad+\int_{\varepsilon}^{r} d s \int_{r-s}^{r+\varepsilon} d t \square+\int_{r}^{2 r+\varepsilon} d s \int_{s-r}^{r+\varepsilon} d t \square \\
=\frac{3}{8}\left(\frac{(\varepsilon-r)^{2}(\varepsilon+5 r)}{6 r^{3}}-\frac{(\varepsilon+r)\left(\varepsilon^{2}+2 \varepsilon r-11 r^{2}\right)}{6 r^{3}}\right)=1,
\end{aligned}
$$

where $\square=3 t^{-2} s^{-2} \theta / 8$.

If we reverse the order of integration in (8), take the limit $r \rightarrow \infty$, and use (B1) and $g(\infty)=1$, we obtain

$$
f(\infty)=1-\frac{8}{3} \pi n \alpha f(\infty),
$$

and therefore $f(\infty)=f_{L}$.

\section{APPENDIX C: CALCULATION OF THE ELECTRONIC CENTER OF MASS AND LOCALIZATION LENGTH WITH PERIODIC BOUNDARY CONDITIONS}

The center of mass of the excess electron and the localization length are defined from the wavefunction $\phi(\mathbf{r})$ as $\langle\phi|\mathbf{r}| \phi\rangle$ and $\sqrt{\langle\phi|\mathbf{r} \cdot \mathbf{r}| \phi\rangle-\langle\phi|\mathbf{r}| \phi\rangle \cdot\langle\phi|\mathbf{r}| \phi\rangle}$, respectively. However, under periodic boundary conditions these quantities are not well defined mathematically even for localized states, and different values are obtained if the origin of the system is chosen so that the boundaries cross a region where the electron has a significant density probability. Following the localization criterion presented in Ref. 5, we have overcome this problem by choosing each origin component at the grid points so that the absolute value of the flux $\Phi$ across the corresponding boundary is minimized, where

$$
\Phi=\int d S \phi \mathbf{n} \cdot \nabla \phi
$$

and $\mathbf{n}$ is a unit vector perpendicular to the boundary surface. This procedure guarantees that wave function has decayed at the boundaries and the localization length is well defined.

\footnotetext{
${ }^{1}$ See, e.g., L. A. Dissado and J. C. Fothergill, Electrical Degradation and Breakdown in Polymers (Peregrinus, London, UK, 1992) and references therein.

${ }^{2}$ N. H. Ge, C. M. Wong, R. L. Lingle, J. D. McNeill, K. J. Gaffney, and C. B. Harris, Science 287, 288 (1998).

${ }^{3}$ S. Serra, S. Iarlori, E. Tosatti, S. Scandolo, M. C. Righi, and G. E. Santoro, Chem. Phys. Lett. 360, 487 (2002).

${ }^{4}$ D. Cubero, N. Quirke, and D. F. Coker, Chem. Phys. Lett. 370, 21 (2003).

${ }^{5}$ D. Cubero, N. Quirke, and D. F. Coker, J. Chem. Phys. 119, 2669 (2003).

${ }^{6}$ M. H. Gutknecht, Acta Numerica (Cambridge U.P., Cambridge, 1997).

${ }^{7}$ W. Smith and T. Forester, J. Mol. Graphics 14, 136 (1996).

${ }^{8}$ K. Drukker, J. Comput. Phys. 153, 225 (1999).

${ }^{9} \mathrm{~J}$. Tully, in Classical and Quantum Dynamics in Condensed Phase Simulations, edited by B. Berne, G. Ciccotti, and D. Coker (World Scientific, Singapore, 1998).

${ }^{10}$ J. Lekner, Phys. Rev. 158, 130 (1967).

${ }^{11}$ B. Space, D. F. Coker, Z. H. Lui, B. J. Berne, and G. Martyna, J. Chem. Phys. 97, 2002 (1992).

${ }^{12}$ N. W. Ashcroft and N. D. Mermin, Solid State Physics (Saunders, Fort Worth, 1976).

${ }^{13}$ See A. L. Shluger and A. M. Stoneham, J. Phys.: Condens. Matter 5, 3049 (1993) and/or J. Appel. Solid Stat. Phys. 21, 193 (1968).

${ }^{14}$ H. C. Andersen, J. Chem. Phys. 72, 2384 (1980).

${ }^{15}$ D. Frenkel and B. Smit, Understanding Molecular Simulation (Academic, San Diego, 2002).

${ }^{16}$ N. F. Mott and E. A. Davis, Electronic Processes in Non-crystalline Materials (Clarendon, Oxford, 1979).

${ }^{17}$ S. F. Parker, J. Chem. Soc., Faraday Trans. 92, 1941 (1996).

${ }^{18}$ A. V. Savin and L. I. Manevitch, Phys. Rev. B 67, 144302 (2003).

${ }^{19}$ D. Cubero, N. Quirke, and D. F. Coker, in preparation.
} 